\title{
Long-term effectiveness of omalizumab treatment in Thai severe asthmatic patients: a real-life experience
}

\author{
Theerasuk Kawamatawong, ${ }_{1}$ Orapan Poachanukoon, ${ }^{2}$ Chalermporn Boonsiri, ${ }^{3}$ Atik Saengasapaviriya, ${ }^{4}$ \\ Chanchai Sittipunt, ${ }^{5}$ Hiroshi Chantaphakul, ${ }^{6}$ Kittipong Maneechotesuwan, ${ }^{7}$ Pintip Ngamchanyaporn, ${ }^{8}$ \\ Kunchit Piyavechviratana, ${ }^{9}$ Praparn Yongjaiyut, ${ }^{7}$ Apichart Khanisap, ${ }^{2}$ Siwasak Juthong, ${ }^{10}$ \\ Warangkana Rithirak, ${ }^{10}$ Prapaporn Pornsuriyasak, ${ }^{1}$ Chaicharn Pothirat, ${ }^{11}$ Watchara Boonsawat ${ }^{12,13}$
}

\begin{abstract}
Background: To evaluate long-term effectiveness of omalizumab in 'real-life' setting of Thai asthmatic patients.

Methods: We conducted multi-center, observational study in severe asthma patients who received omalizumab in Thailand. Outcomes were asthma exacerbation (hospitalization and ER visit), asthma control test (ACT), and daily ICS dose. Data were evaluated at baseline, 16 Week, and 52 Week.

Results: A total of 78 patients received omalizumab treatment (average duration 16.9 months with range 16 weeks-2 years). The mean annualized rate of exacerbations was reduced from baseline (3.79) at Week 16 (3.54) and Week 52 (1.16), $(\mathrm{p}<0.05)$, respectively. The mean hospitalization rate was reduced from 0.49 in previous year to 0.15 at Week 16 and 0.19 at Week 52. A reduction in ER visit rates was observed at Week $16(0.15)$ and Week $52(0.97)$ respectively from baseline (1.44) $(\mathrm{p}<0.05)$. The ACT score increased from 15.4 at baseline to 20.6 at Week $16(\mathrm{p}<0.001)$ and increased to 21.5 at Week $52(\mathrm{p}<0.001)$. The number of patients with controlled asthma (ACT $\geq 20)$ increased from 16 of 51 at baseline to 32 of 45 at Week 16 and 25 of 32 at week 52, respectively. The median daily dose of ICS equivalent to fluticasone was reduced from baseline $680 \mathrm{mcg}$ to $500 \mathrm{mcg}$ at Week 52. In all, 22 patients discontinued omalizumab after 1 year. Six patients who discontinued omalizumab were restarted due to relapse of symptoms.
\end{abstract}

Conclusions: These data confirms the effectiveness of one-year duration of omalizumab treatment in Thai severe asthmatic patients. Furthermore, $27 \%$ of patients who discontinued treatment required restarting due to relapse of symptoms.

Keywords: Omalizumab, Severe Asthma, Effectiveness, Long-term, Real life

From:

Division of Pulmonary and Critical Care, Department of Medicine, Faculty of Medicine, Ramathibodi Hospital, Mahidol University, Bangkok, Thailand.

Faculty of Medicine, Thammasart University, Pathumthani, Thailand.

Directorate of Medical Service, Royal Thai Air Force,

Bhumipol Hospital, Bangkok Thailand

Division of Immunology, Department of Medicine, Phramongkutklao Hospital, Bangkok, Thailand.

Division of Pulmonary and Pulmonary Critical Care, Department of Medicine, Faculty of Medicine,

The King Chulalongkorn Memorial Hospital,

Chulalongkorn University, Bangkok, Thailand.

6 Division of Allergy and Immunology, Department of Medicine, Faculty of Medicine, the King Chulalongkorn Memorial Hospital, Chulalongkorn University, Bangkok, Thailand.

Division of Pulmonary and Pulmonary Critical Care,

Department of Medicine, Faculty of Medicine, Mahidol University,

Siriraj Hospital, Bangkok, Thailand.

8 Division of Allergy, Immunology, and Rheumatology,

Department of Medicine, Faculty of Medicine, Ramathibodi Hospital, Mahidol University, Bangkok, Thailand
9 Division of Pulmonary and Critical Care, Department of Medicine, Phramongkutklao Hospital, Bangkok, Thailand.

${ }^{10}$ Division of Respiratory and Respiratory Critical Care Medicine, Department of Medicine, Faculty of Medicine, Prince of Songkla University, Songkla, Thailand.

${ }^{11}$ Division of Pulmonary and Critical Care, Department of Medicine, Faculty of Medicine,, Chiang Mai University, Chiang Mai, Thailand.

${ }^{12}$ Division of Pulmonary Medicine, Department of Medicine, Faculty of Medicine, Khon Kaen University, Khon Kaen, Thailand

${ }^{13}$ Thai Asthma Council

Corresponding author:

Orapan Poachanukoon

Faculty of Medicine, Thammasart University, Khlongnueng sub-district, Khlongluang district,

Pathumthani, Thailand 12120

E-mail: orapanpoachanukoon@yahoo.com 


\section{Background}

Asthma is one of the major public health concerns in Thailand. In a survey study conducted in 2004, the burden of asthma was high, with $14.8 \%$ of respondents being hospitalized for their asthma from the past year and 9\% reporting severe persistent asthma. ${ }^{1}$ Asthma severity is classified based on the intensity of treatment required to achieve good control of symptoms. In general, patients with mild to moderate persistent asthma can be controlled with low-dose inhaled corticosteroids (ICS) plus a long-acting $\beta_{2}$ agonist (LABA) whereas patients with severe persistent asthma are often not well controlled despite treating with high dose ICS-LABA combination. Inadequately controlled patients are prone to recurrent exacerbations, hospitalization and increased mortality. Often, oral corticosteroids (OCS) are often additionally administered to suppress airway inflammation and improve symptoms; however, long-term use of OCS is associated with substantial side effects, including diabetes, osteoporosis and cataract formation, placing a major burden on patients and healthcare resources. ${ }^{2-5}$

Omalizumab, a humanized anti-immunoglobulin E (IgE) monoclonal antibody, was approved in Thailand in 2006 as an add-on therapy for severe allergic asthma, inadequately controlled with high dose ICS-LABA combination therapy. Omalizumab has been studied in several randomized controlled trials and observational real-life clinical practices involving different ethnic groups. Omalizumab has been shown to reduce asthma exacerbations and hospitalization rates, as well as requirements for oral corticosteroids, in patients with allergic asthma. ${ }^{6-11}$ The clinical benefits of omalizumab in "real-world" settings has been demonstrated consistent with the results from clinical controlled trials, providing reassurance of the improvements in outcomes in patients with uncontrolled persistent allergic asthma. ${ }^{12-14}$ The long term observational studies of omalizumab have proven its efficacy in terms of sustained asthma control without rebound effect on clinical or immunological parameters. ${ }^{15-17}$ In addition, the safety of omalizumab has been confirmed in post-marketing surveillance. ${ }^{18,19}$

However, omalizumab is considered a high cost medication in Thailand with a lack of evidence to prove its clinical benefits among Thai patients suffered from severe persistent allergic asthma. The Omalizumab (Xolair) retrospective studY and reGistry in Thai asthmatic patiENts study (OXYGEN) was therefore initiated in 2009 to address the need to generate data specific to Thai patients and assess whether omalizumab has similar efficacy in this patient population. Patients with severe allergic persistent asthma treated with omalizumab both continuously and intermittently were included in this registry. The results of this study confirm the long-term effectiveness of omalizumab in a real-life setting among severe asthma patients in Thailand.

\section{Methods \\ Study Design and Patient Population}

We conducted a multi-center, cross-sectional observational study in allergy and pulmonology clinics from 10 medical centers across Thailand. Data were collected from paper and electronic hospital medical record between November 2009 and November 2013. A total of 78 patients were identified, including patients from Thammasat University (15 patients), Ramahibodi Hospital, Mahidol University (20 patients), Phramongkutklao Hospital (13 patients), Bhumiphol hospital (13 patients), the King Chulalongkorn Memorial Hospital, Chulalongkorn University (8 patients), Prince of Songkla University Hospital (4 patients), Siriraj Hospital, Mahidol University (3 patients) and Chiang Mai University (2 patients). All patients included in the study were confirmed to have received omalizumab treatment according to routine clinical practice based on the approved indication in Thailand as an add-on therapy for treatment of severe allergic asthma in patients inadequately controlled despite the use of high-dose inhaled corticosteriod (ICS) plus long-acting $\beta 2$-agonists (LABA). Only patient's with age $\geq 6$ years at time of omalizumab initiation, with a documented diagnosis of inadequately controlled severe persistent allergic asthma, with a documentation of asthma exacerbations prior to omalizumab initiation, and in whom omalizumab had been used for at least 16 weeks at time of enrollment were enrolled in the study. Patients with duration of omalizumab treatment less than 16 weeks were excluded, as the response to treatment is recommended to be evaluated at least 16 weeks after initiation of treatment. This study was approved by Institutional Review Board/Ethic Committees in each study center.

\section{Data collection}

The investigators from each center were asked to identify patients who received omalizumab and fulfilled the study criteria from their medical centers' patient database and review and collect data from the patients' medical records. Data collection included patient demographics, omalizumab dosing, concomitant asthma medications, exacerbations, hospital admissions, emergency visits, and Asthma Control Test (ACT) scores. Asthma exacerbations were defined as an increase in symptoms requiring treatment with oral corticosteroids (OCS) or doubling of the ICS dose. Data were collected at baseline (at the initiation of omalizumab), Week 16 (Month 4), and up to Week 52 (Year 1) after omalizumab treatment. Data beyond 1 year of treatment collected for exploratory purposes. The overall duration of omalizumab treatment up to the time of data collection was recorded. Data regarding treatment discontinuation and restart of omalizumab treatment were collected to determine treatment duration, reason of discontinuation, number of symptoms at the time of relapse and the time interval before restart of treatment. Asthma relapses after treatment discontinuation were defined as asthma exacerbations requiring OCS or doubling of ICS dose and/or persistent worsening or increase in asthma symptoms as judged clinically significant by investigators.

\section{Omalizumab treatment and concomitant medications}

Omalizumab doses were calculated according to the omalizumab dosing table based on individual patient's baseline IgE level and body weight. Omalizumab in Thailand is approved as an add-on therapy for the treatment of severe allergic asthma in patients age 6 or above who are inadequately controlled despite high dose ICS-LABA therapy. ${ }^{20}$ 
Omalizumab was administered by subcutaneous injection every 2 or 4 weeks. There were no restrictions in use of concomitant asthma medications. The medications used, including controller medications, oral corticosteroids and allergic rhinitis drugs, were collected.

\section{Effectiveness Outcomes}

The primary purpose of this study was to evaluate the long-term effectiveness of omalizumab as an add-on therapy in local 'real-life' setting in Thai asthmatic patients. The main effectiveness outcomes were asthma exacerbation rate, hospitalization rate, ER visit rate, level of asthma control (ACT score), and daily ICS dose evaluated at baseline, Week 16, and Week 52. The rates of exacerbation, hospitalization, and ER visits were evaluated as the change in annualized rate from baseline. The level of asthma control was evaluated by change from baseline in ACT score and the change in proportion of patients with controlled and uncontrolled asthma as defined by ACT score. The daily ICS dose was converted into fluticasone-equivalent dose and then evaluated as a reduction from baseline at Week 16 and Week 52. Other concomitant asthma medications were evaluated as the reduction in percentage of patients with use of the medications as compared with baseline. The exploratory outcomes of this study were assessment of relapse rates and the frequency of restarting of treatment after discontinuation.

\section{Statistical Analysis}

The Stata version 14 (StataCorp, College Station, TX) was used for the entire analysis. A p-value $<0.05$ was used to indicate a statistical significant difference, using Wilcoxon Signed Ranks test. Descriptive data were expressed as mean + standard deviation (SD) for continuous variables, and as number and percentage for categorical variables.

\section{Results}

\section{Patient Characteristics}

A total of 78 omalizumab-treated patients were reviewed. Table 1 illustrates the baseline patient demographic and clinical characteristics of patients. The median age of patients was 58.5 (50.7-68.0) years, and the median duration of asthma was 10 years. The median baseline $\mathrm{FEV}_{1} \%$ predicted was 67.45 (54.0-89.3), and the median PEFR was 310 (211-684) L/min. The median baseline total serum IgE was 257 (97-544) IU/mL. The median monthly omalizumab dose was 300 (150-1200) $\mathrm{mg}$. The average duration of omalizumab use was 16.9 months with range of 16 weeks to 2 years. Sixty-one patients out of 78 patients (78.2\%) had documented allergic test reports, either skin prick test or sIgE (RAST), and 51 of which (83.6\%) had positive allergic test results. The reports of the remaining 17 patients were missing. All patients were taking an inhaled corticosteroid plus inhaled long-acting $\beta 2$-agonist; most patients were taking these as a fixed-dose combination. The median daily fluticasone-equivalent ICS dose was $680 \mathrm{mcg}$. Maintenance OCS were prescribed in $26.9 \%$ of patients. At baseline, $69.2 \%$ of patients were taking a leukotriene antagonists and $30.8 \%$ were taking an anti-histamine. Interestingly, $44.9 \%$ of patients were prescribed theophylline as their maintenance asthma medication. The most common concomitant allergic condition was allergic rhinitis (88.5\%). The patient demographic and baseline characteristics are summarized in Table $\mathbf{1}$.

Table 1. Patient demographic and baseline characteristics.

\begin{tabular}{|c|c|}
\hline Parameters & $\begin{array}{l}\text { Study population } \\
\qquad(\mathrm{n}=78)\end{array}$ \\
\hline Gender (n, F/M) & $51 / 27$ \\
\hline Age, median and IQR (years) & $58.5(50.7-68.0)$ \\
\hline $12-17$ years, $n(\%)$ & $8(10.4 \%)$ \\
\hline$>18$ years, $\mathrm{n}(\%)$ & $69(89.6 \%)$ \\
\hline Body weight, median and IQR (kg) & $62.7(53.0-68.4)$ \\
\hline Duration of asthma, median and IQR (years) & $10(2.75-23.25)$ \\
\hline Baseline total IgE, median and IQR (IU/mL) & $257(97-544)$ \\
\hline Proportion of patients with allergic tests & $61(78.2 \%)$ \\
\hline \multicolumn{2}{|l|}{ Positive allergic tests, $\mathrm{n}(\%)$} \\
\hline (aeroallergen: house dust mite, cockroach, cat) & $51(65.4 \%)$ \\
\hline $\begin{array}{l}\text { Baseline } \mathrm{FEV}_{1} \% \text { predicted, median and IQR } \\
\text { (\% predicted) }\end{array}$ & $67.5(54.0-89.3)$ \\
\hline Baseline PEFR, median and IQR (L/min) & $310(211-684)$ \\
\hline Concomitant allergic diseases, $\mathrm{n}(\%)$ & $78(100 \%)$ \\
\hline Allergic rhinitis & $69(88.5 \%)$ \\
\hline Allergic conjunctivitis & $9(11.5 \%)$ \\
\hline Atopic dermatitis, eczema & $6(7.7 \%)$ \\
\hline Urticaria & $4(5.1 \%)$ \\
\hline Omalizumab dose, median and IQR (mg/month) & $300(150-1200)$ \\
\hline $\begin{array}{l}\text { Daily ICS dose at baseline, median and IQR } \\
\text { (mcg/day) (mcg equivalent to fluticasone per day) }\end{array}$ & $680(160-2000)$ \\
\hline
\end{tabular}

Abbreviation: IQR; interquartile range, IgE; immunoglobulin $\mathrm{E}, \mathrm{FEV}_{1}$; forced expiratory volume in the $1^{\text {st }}$ second, PEFR; peak expiratory flow rate, and ICS; inhaled corticosteroi

Table 2. Mean annualized rate of asthma exacerbation, hospitalization, and emergency visit at baseline, Week 16, and Week 52.

\begin{tabular}{lccc}
\hline \multicolumn{1}{|c}{ Annualized rate } & $\begin{array}{c}\text { Baseline } \\
(n=78)\end{array}$ & $\begin{array}{c}\text { Week 16 } \\
(n=78)\end{array}$ & $\begin{array}{c}\text { Week 52 } \\
(n=62)\end{array}$ \\
\hline Asthma exacerbation rate & 3.79 & $3.54^{\mathrm{b}}$ & $1.16^{\mathrm{c}}$ \\
\hline Hospitalization rate & 0.49 & $0.15^{\mathrm{b}}$ & $0.19^{\mathrm{c}}$ \\
\hline Emergency visit rate & 1.44 & $0.15^{\mathrm{b}}$ & $0.97^{\mathrm{c}}$ \\
\hline
\end{tabular}

a Annualized asthma exacerbation, hospitalization and ER visit rates during treatment follow-up period compared with that of baseline (for p-value, statistically significant difference from baseline using Wilcoxon test).

${ }^{\mathrm{b}} p<0.05$, baseline vs week 16

${ }^{c} p<0.05$, baseline vs week 52 


\section{Reduction in Asthma Exacerbations, Hospitalization, and ER Visit Rate}

The mean annualized rate of overall asthma exacerbations requiring an increased systemic corticosteroids and/or doubling dose of ICS was significantly reduced from 3.79 in the year prior to treatment to 3.54 at Week $16(p<0.05)$ and 1.16 at Week $52(p<0.05)$. The mean hospitalization rate was also reduced from 0.49 in previous year to 0.15 at Week 16 and 0.19 at Week 52. Similarly, a significant reduction in ER visit rate from baseline (1.44) was observed at Week $16(0.15)$, and Week 52 (0.97) (both $p<0.05)$.

\section{Improvement in Level of Asthma Control}

The level of asthma control was evaluated by ACT (Asthma Control Test) score. Only 51 patients had their ACT score documented. Summary of patient deposition was shown (Figure 1). Among these, ACT was increased from 15.4 at baseline to 20.6 at Week $16(32.4 \%$ improvement, $\mathrm{p}<0.01)$ and increased further to 21.5 at Week 52 (28.7\% improvement, $\mathrm{p}<0.001$ ) Figure 2). For comparative purposes, patients were separated into 2 categories according to their asthma control as defined by ACT score - patients with controlled asthma $(\mathrm{ACT} \geq 20)$ and patients with uncontrolled asthma $(\mathrm{ACT}<20)$. At baseline, a high percentage of patients (68.6\%) was uncontrolled. After 16 weeks of treatment; the proportion of patients with controlled asthma increased from $31.4 \%$ at baseline to $71.1 \%$, corresponding to a decrease in the proportion of patients with uncontrolled asthma to $28.9 \%$. This improvement persisted until 52 weeks of treatment as demonstrated with $78.1 \%$ of patients with controlled asthma and $21.8 \%$ of patients with uncontrolled asthma at this time point.

\section{Reduction in Daily ICS Dose and Other Concomitant Asthma Medications}

The median daily dose of ICS was slightly reduced from baseline dose of $680 \mathrm{mcg}$ equivalent to fluticasone per day to
$500 \mathrm{mcg}$ per day at Week 52 after treatment as shown in Table 3. As for other concomitant asthma medications, the proportion of patients using maintenance oral corticosteroids decreased from $26.9 \%$ (21/78 patients) at baseline to $14.1 \%$ (11/78 patients) at Week 16 and $6.4 \%(4 / 62)$ at Week 52 (Table 3). Similarly, the proportion of patients receiving concomitant treatment with LTRA, theophylline, and anti-histamine was also reduced during omalizumab treatment.

Table 3. Changes in daily dose of inhaled corticosteroid (mcg equivalent to fluticasone per day) and number of patients on concomitant medications at each time point.

\begin{tabular}{lccc}
\hline $\begin{array}{l}\text { Asthma and concomitant } \\
\text { mediations }\end{array}$ & $\begin{array}{c}\text { Baseline } \\
(n=78)\end{array}$ & $\begin{array}{c}\text { Week 16 } \\
(n=78)\end{array}$ & $\begin{array}{c}\text { Week 52 } \\
(n=62)\end{array}$ \\
\hline $\begin{array}{l}\text { Mean ICS daily dose } \\
\text { (mcg equivalent to } \\
\text { fluticasone per day) }\end{array}$ & 591.9 & $485.6^{\mathrm{a}}$ & $356.5^{\mathrm{b}}$ \\
\hline $\begin{array}{l}\text { Median ICS daily dose } \\
\text { IQR) } \\
\text { (mcg equivalent to } \\
\text { fluticasone per day) }\end{array}$ & 680 & 840 & $500^{\mathrm{b}}$ \\
\hline $\begin{array}{l}\text { Number of patients on } \\
\text { concomitant medications, } \\
\text { n (\%) }\end{array}$ & $\begin{array}{c}(n=78) \\
\text { Baseline }\end{array}$ & $\begin{array}{c}\text { Week 16 } \\
(n=78)\end{array}$ & $\begin{array}{c}\text { Week 52 } \\
(n=62)\end{array}$ \\
\hline $\begin{array}{l}\text { Oral corticosteroids } \\
\text { LTRA }\end{array}$ & $21(26.9 \%)$ & $11(14.1 \%)$ & $4(6.4 \%)$ \\
\hline $\begin{array}{l}\text { Theophylline } \\
\text { Anti-histamine }\end{array}$ & $54(69.2 \%)$ & $44(56.4 \%)$ & $22(34.9 \%)$ \\
\hline \begin{tabular}{l} 
Nasal corticosteroid \\
\hline
\end{tabular} & $35(44.9 \%)$ & $25(32.1 \%)$ & $15(23.8 \%)$ \\
\hline
\end{tabular}

${ }^{a} p<0.05$, baseline vs week 16

${ }^{b} p<0.05$, baseline vs week 52

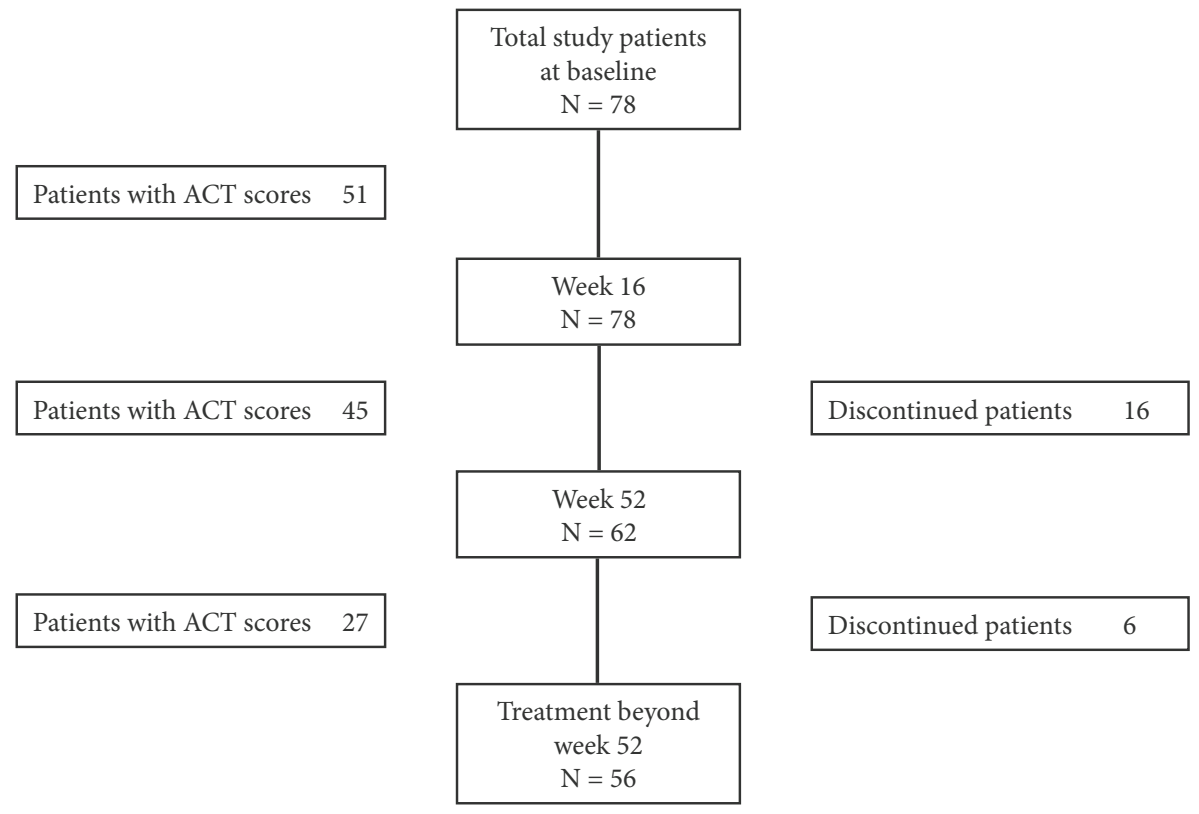

Figure 1. Summary of patient deposition 


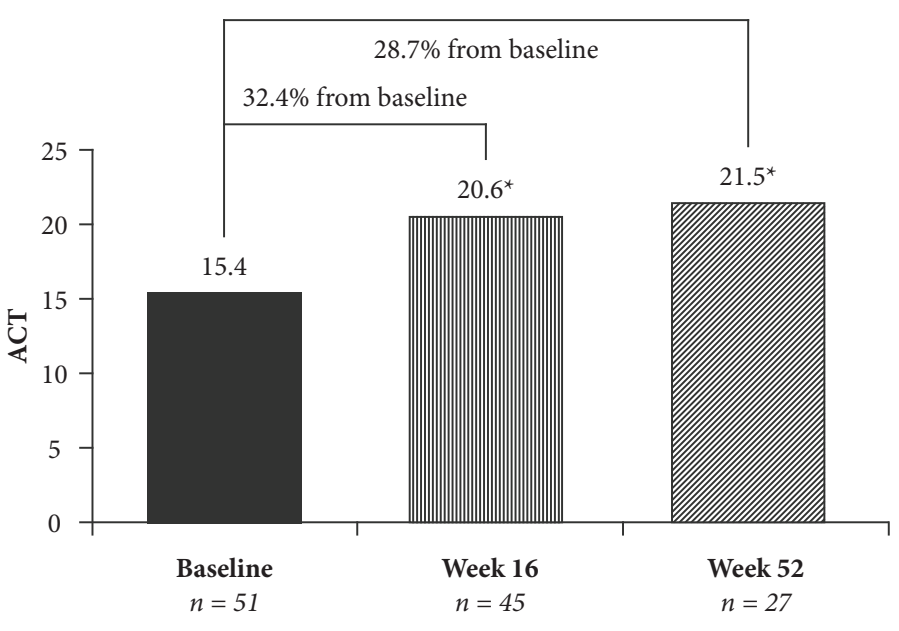

${ }^{*} p<0.001$ vs baseline

Figure 2. Level of asthma control improvement ACT scores and percentage change from baseline to week 16 and week 52 .

\section{Treatment Discontinuation and Restart of Omalizumab Treat- ment}

In all, 62 patients (79.4\%) continued omalizumab treatment for at least 1 year. Of the 22 patients who discontinued omalizumab, the median treatment duration was 12 (from 4 to 48 ) months. The most common reasons for discontinuation of treatment were patient desire (50\%), loss to follow up (13.6\%) and physician desire (9.15\%). Six patients (27\%) who discontinued omalizumab treatment were restarted due to a relapse of symptoms. Among patients who restarted omalizumab, the mean dose of omalizuamb was $300 \pm 164.3 \mathrm{mg}$, the mean duration of treatment prior to discontinuation was $9.7 \pm 6.3$ months and the mean duration of omalizumab discontinuation was $6.5 \pm 3.4$ months. The mean serum total IgE before restarting treatment was $355.7 \pm 130.2 \mathrm{IU} / \mathrm{ml}$. There were no serious adverse effects related to omalizumab treatment recorded.

\section{Discussion}

Despite the fact that there have been several randomized controlled trials demonstrating the clinical effectiveness of omalizumab in severe allergic asthma, real world studies for documenting effectiveness are also important. The advantages of real-life studies are maximizing external validity, increasing generalizability and being preferable evidence within healthcare systems. ${ }^{21}$

The results of our study included both adults and children with severe asthma. In addition, this study is the first to provide real world evidence of the effectiveness of omalizumab in Southeast Asian region. The average duration of omalizumab treatment in Thai asthmatics was 16.9 months (16 weeks-2 years) due to the regulation for reimbursement in the healthcare system in Thailand. Omalizumab effectiveness in our study was assessed in terms of clinically significant asthma exacerbation reduction rate and improvement of asthma control level (ACT) score similar to a previously reported pooled analysis comprising seven randomized studies. ${ }^{22}$ However our asthmatic patients were older, had more severe airflow obstruction and had a higher baseline total serum IgE. ${ }^{23}$
Our patient baseline characteristics were similar to those in a previously reported real world study conducted in France. ${ }^{24}$

Outcomes of asthma control can be measured by assessment of current symptom scores, frequency of asthma exacerbations and oral corticosteroid usage. ${ }^{25}$ We found that a composite symptom score of asthma control, the asthma control test (ACT) score, was significantly improved at the $16^{\text {th }}$ week of treatment in this study. However, there was no further improvement after the $52^{\text {nd }}$ week of therapy. The improving of asthma composite score was similar to previous real world studies. ${ }^{12,13,26,27}$

More significant improvement of asthma outcome was found in our study using exacerbation frequency and reduction of oral corticosteroid usage. These findings support that omalizumab effectiveness was similar to the previous real world studies. Treatment with omalizumab has been associated with a reduction or discontinuation of added on oral corticosteroid required for controlling asthma. ${ }^{11,12,24}$ In our study, omalizumab treatment was also associated with a trend toward a reduction in the dose of inhaled corticosteroid, although this trend did not reach statistical significance. In a much larger prospective observational cohort study of moderate-to-severe allergic asthma, omalizumab therapy was associated with a reduction in doses of ICS, SABA, and leukotriene modifiers over 2 years in a "real-world" setting. ${ }^{14}$

The recommended duration of omalizumab treatment has never been clearly established. Nevertheless, the result of a long term study of omalizumab treatment (7 years) demonstrated the additional benefit of extended treatment duration by reducing exacerbation rate. ${ }^{17}$ Apart from sustained clinical efficacy, long term omalizumab treatment has also been associated with a sustained reduction of in vitro basophil allergen threshold sensitivity that persists beyond discontinuation of treatment. ${ }^{15,16}$ Current Thai asthma guidelines limit long term treatment with omalizumab despite clinical evidence of improvement. For this reason, approximately one-third of our patients discontinued omalizumab injections. Twenty-seven percent of patients who discontinued treatment developed clinical relapse determined by the presence of asthma exacerbation requiring systemic corticosteroid that resulted in re-starting of omalizumab treatment. The asthmatic relapse developed after 6.5 month of discontinued treatment. This finding emphasizes the long term benefit of continuation of omalizumab treatment in a real life study.

The duration of follow up after discontinuation of omalizumab treatment is essential for determining the clinical relapse rate. In our study, the duration of follow up of patients who discontinued omalizumab was shorter than in the French study, and this reason may explain why the proportion of patients who developed clinical relapse was lower in our study compared to the French real world study. ${ }^{24}$ The limitations of our real world study are incomplete data of asthma outcome measurement in the follow up period. In addition, approximately $20 \%$ of patients who received omalizumab were either current or former smokers, and we could not distinguish evere asthma from either COPD or asthma COPD overlap syndrome (ACOS) in this subset of patients. ${ }^{28,30}$ However, the presence of fixed airflow obstruction, the long duration of diagnosed asthma, the high serum total IgE and skin prick test positivity to common aeroallergen in the majority of these 
patients are compatible with the ACOS definition. The study for comparing efficacy of adding anticholinergic or adding omalizumab to ICS/LABA in these patients with ACOS are needed.

\section{References}

1. Boonsawat W, Charoenphan P, Kiatboonsri S, et al. Survey of asthma control in Thailand. Respirology. 2004; 9: 373-378.

2. Bousquet J, Wenzel S, Holgate S, Lumry W, Freeman P, Fox H. Predicting response to omalizumab, an anti-IgE antibody, in patients with allergic asthma. Chest. 2004; 125:1378-1386.

3. Fardet L, Flahault A, Kettaneh A, et al. Corticosteroid-induced clinical adverse events: frequency, risk factors and patient's opinion. Br J Dermatol. 2007;157:142-148

4. Manson SC, Brown RE, Cerulli A, Vidaurre CF. The cumulative burden of oral corticosteroid side effects and the economic implications of steroid use. Respir Med 2009;103:975-994.

5. Walsh LJ, Wong CA, Oborne J, Cooper S, Lewis SA, Pringle M et al. Adverse effects of oral corticosteroids in relation to dose in patients with lung disease. Thorax. 2001;56:279-284.

6. Holgate ST, Chuchalin AG, Hebert J, Lötvall J, Persson GB, Chung KF et al. Efficacy and safety of a recombinant anti-immunoglobulin E antibody (omalizumab) in severe allergic asthma. Clin Exp Allergy. 2004;34:632-638.

7. Lafeuille MH, Dean J, Zhang J, Duh MS, Gorsh B, Lefebvre P. Impact of omalizumab on emergency-department visits, hospitalizations, and corticosteroid use among patients with uncontrolled asthma. Ann Allergy Asthma Immunol. 2012;109:59-64.

8. Soler M, Matz J, Townley R, et al. The anti-IgE antibody omalizumab reduces exacerbations and steroid requirement in allergic asthmatics. Eur Respir J. 2001;18:254-261.

9. Humbert M, Beasley R, Ayres J, Slavin R, Hébert J, Bousquet J, et al. Benefits of omalizumab as add-on therapy in patients with severe persistent asthma who are inadequately controlled despite best available therapy (GINA 2002 step 4 treatment): INNOVATE. Allergy 2005;60:309-316.

10. Hanania NA, Alpan O, Hamilos DL, Condemi JJ, Reyes-Rivera I, Zhu J, et al. Omalizumab in severe allergic asthma inadequately controlled with standard therapy: a randomized trial. Ann Intern Med. 2011;154:573-582.

11. Pelaia G1, Gallelli L, Romeo P, Renda T, Busceti MT, Proietto A, et al. Omalizumab decreases exacerbation frequency, oral intake of corticosteroids and peripheral blood eosinophils in atopic patients with uncontrolled asthma. Int J Clin Pharmacol Ther.2011;49:713-721.

12. Braunstahl GJ, Chen CW, Maykut R, Georgiou P, Peachey G, Bruce J. The eXpeRience registry: the 'real-world' effectiveness of omalizumab in allergic asthma. Respir Med. 2013;107:1141-1151.

13. Tzortzaki EG1, Georgiou A, Kampas D, Lemessios M, Markatos M, Adamidi $\mathrm{T}$, et al. Long-term omalizumab treatment in severe allergic asthma: the South-Eastern Mediterranean "real-life" experience. Pulm Pharmacol Ther. 2012;25:77-82.

14. Chen H, Eisner MD, Haselkorn T, Trzaskoma B. Concomitant asthma medications in moderate-to-severe allergic asthma treated with omalizumab. Respir Med. 2013;107:60-67.
15. Nopp A, Johansson SG, Ankerst J, Palmqvist M, Oman H. CD-sens and clinical changes during withdrawal of Xolair after 6 years of treatment. Allergy. 2007;62:1175-1181

16. Nopp A, Johansson SG, Adedoyin J, Ankerst J, Palmqvist M, Oman H. After 6 years with Xolair; a 3-year withdrawal follow-up. Allergy. 2010;65:56-60.

17. Pace E, Ferraro M, Bruno A, Chiappara G, Bousquet J, Gjomarkaj M. Clinical benefits of 7 years of treatment with omalizumab in severe uncontrolled asthmatics. J Asthma. 2011;48:387-392.

18. Corren J. Efficacy and safety of budesonide and formoterol in asthma. Hosp Pract. 2009;37:162-163.

19. Ohta K, Miyamoto T, Amagasaki T, Yamamoto M. Efficacy and safety of omalizumab in an Asian population with moderate-to-severe persistent asthma. Respirology. 2009;14:1156-1165.

20. Price D. The use of omalizumab in asthma. Prim Care Respir J. 2008;17: 62-72.

21. Price D, Chisholm A, van der Molen T, Roche N, Hillyer EV, Bousquet J. Reassessing the evidence hierarchy in asthma: evaluating comparative effectiveness. Curr Allergy Asthma Rep. 2011;11:526-538.

22. Bousquet J, Cabrera P, Berkman N, Buhl R, Holgate S, Wenzel S, et al. The effect of treatment with omalizumab, an anti-IgE antibody, on asthma exacerbations and emergency medical visits in patients with severe persistent asthma. Allergy. 2005;60:302-308.

23. Busse WW, Massanari M, Kianifard F, Geba GP. Effect of omalizumab on the need for rescue systemic corticosteroid treatment in patients with moderate-to-severe persistent IgE-mediated allergic asthma: a pooled analysis. Curr Med Res Opin. 2007;23:2379-2386.

24. Molimard M, Mala L, Bourdeix I, Le Gros V. Observational study in severe asthmatic patients after discontinuation of omalizumab for good asthma control. Respir Med. 2014;108:571-576.

25. Reddel HK, Taylor DR, Bateman ED, Boulet LP, Boushey HA, Busse WW, et al. An official American Thoracic Society/European Respiratory Society statement: asthma control and exacerbations: standardizing endpoints for clinical asthma trials and clinical practice. Am J Respir Crit Care Med. 2009;180:59-99.

26. Vennera Mdel C, Pérez De Llano L, Bardagí S, Ausin P, Sanjuas C, González $\mathrm{H}$, et al. Omalizumab therapy in severe asthma: experience from the Spanish registry--some new approaches. J Asthma. 2012;49:416-422.

27. Eisner MD, Zazzali JL, Miller MK, Bradley MS, Schatz M. Longitudinal changes in asthma control with omalizumab: 2-year interim data from the EXCELS Study. J Asthma. 2012;49:642-648.

28. Louie S, Zeki AA, Schivo M, Chan AL, Yoneda KY, Avdalovic M, et al The asthma-chronic obstructive pulmonary disease overlap syndrome: pharmacotherapeutic considerations. Expert Rev Clin Pharmacol. 2013;6: 197-219.

29. Soler-Cataluña JJ1, Cosío B, Izquierdo JL, López-Campos JL, Marín JM, Agüero R, et al. Consensus document on the overlap phenotype COPD-asthma in COPD. Arch Bronconeumol. 2012;48:331-337.

30. Zeki AA, Schivo M, Chan A, Albertson TE, Louie S. The Asthma-COPD Overlap Syndrome: A Common Clinical Problem in the Elderly. J Allergy. 2011;2011:861926 